\title{
Silicon-Based Mode Converter and Demultiplexer for Wavelength Division Multiplexing Transmission by Using Multimode Interference Couplers
}

\author{
Yong Zhou, Junqiang Sun* \\ National Laboratory for Optoelectronics, School of Optical and Electronic Information, Huazhong University of Science and \\ Technology, Wuhan, China \\ Email: *jqsun@hust.edu.cn
}

How to cite this paper: Zhou, Y. and Sun, J.Q. (2020) Silicon-Based Mode Converter and Demultiplexer for Wavelength Division Multiplexing Transmission by Using Multimode Interference Couplers. Optics and Photonics Journal, 10, 117-124.

https://doi.org/10.4236/opj.2020.106012

Received: April 26, 2020

Accepted: June 26, 2020

Published: June 29, 2020

\begin{abstract}
We present a mode converter and demultiplexer structure for wavelength division multiplexing (WDM) transmission by employing multimode interference (MMI) on Silicon-on-Insulator (SOI) platform. The mode converter and demultiplexer have a compact size of less than $2.7 \mu \mathrm{m} \times 43.7 \mu \mathrm{m}$. Moreover, the crosstalk between neighboring wavelength channel within $\mathrm{C}$ band (1530 nm to $1570 \mathrm{~nm}$ ) can be reduced by utilizing the tapered phase shifter cascaded with MMI. The simulated results indicate that this structure has a low insertion loss of less than $1 \mathrm{~dB}$, a low crosstalk of better than $-15 \mathrm{~dB}$ and a relatively high fabrication tolerance of $\sim 10 \mathrm{~nm}$. Such structure may find many potential applications in silicon photonic integrated devices.
\end{abstract}

\section{Keywords}

Multimode Interference, Wavelength Division Multiplexing, Mode Converter, Silicon-on-Insulator

\section{Introduction}

Silicon-based on-chip optical interconnect has become an emerging technology for its unique advantages of high level of integration. Recently, research work to further increase the transmission capacity of wavelength division multiplexing (WDM) system has become a hot spot in the chip-scale optical communication systems. However, the capacity of WDM is limited by the small bandwidth of fiber amplifier and the low launched power due to fiber nonlinearity. To further 
increase the optical transmission capacity, various kinds of methods have been proposed, such as polarization multiplexing (PDM) and mode multiplexing (MDM) technologies [1]. In addition, WDM interconnections which are compatible with MDM are achieved by using different types of mode de/multiplexer (DE/MUX), which are based on asymmetrical directional couplers (ADC) [2], adiabatic couplers [3] and asymmetrical Y-junctions [4] [5]. However, the ADC structure requires additional phase section to realize mode conversion, leading to a limited fabrication tolerance. The adiabatic couplers can reach a low crosstalk, but at the expense of large footprints. The asymmetrical Y-junction has a small footprint, however, the relatively high insertion loss restricts its applications. In contrast, the multimode interference (MMI) combines the advantages of compact size, low crosstalk, large optical bandwidth, and large fabrication tolerance [6] [7]; thus, it may become a good scheme to achieve high-capacity WDM interconnections.

In this paper, we report a new silicon-based mode converter and demultiplexer structure by employing MMI and tapered phase shifter. As the wavelength is increased, the phase shift induced by tapered phase shifter becomes smaller while the phase shift introduced by MMI becomes larger. Therefore, combing the MMI with the tapered phase shifter, the crosstalk between adjacent wavelengths channels will be reduced substantially. The insertion loss and crosstalk of the presented device are simulated by using 3D finite-different time domain (FDTD) method. The simulated results show the total device has a low insertion loss of less than $1 \mathrm{~dB}$, a crosstalk of better than $-15 \mathrm{~dB}$, and the footprint of $\sim 2.7$ $\mu \mathrm{m} \times 43.7 \mu \mathrm{m}$ (except the length of input/output).

\section{Principle and Device Design}

The structure of MMI based on the self-image principle has been proposed by Lucas B. Soldano [8], and the input image reproduced as mirror or direct images at the period intervals along the propagation direction. $L_{\pi}$ is defined as the beat length of the two lower-order modes:

$$
L_{\pi}=\frac{\pi}{\beta_{0}-\beta_{1}} \cong \frac{4 n_{r} W_{e}^{2}}{3 \lambda_{0}}
$$

where $\beta$ represents the propagation constant of optical mode, $n_{r}$ and $W_{e}$ is the effective reflective index and the width of MMI for wavelength in vacuum of $\lambda_{0}$, respectively.

We propose a mode converter and demultiplexer structure for WDM transmission by employing the principle of MMI, and the wavelength signals are multiplexed into a mode converter and multiplexer structure, then transmitted through the symmetric structure and demultiplexed into the date channel respectively. The mode converter and demultiplexer structure consists of three parts, a symmetric interference MMI coupler (MMI1) as a mode converter, a tapered $\pi / 2$ phase shifter and a $3 \mathrm{~dB}$ general interference MMI coupler (MMI2) as illustrated in Figure 1 . The length of MMI1 and MMI2 are $3 L_{\pi} / 4$ and $3 L_{\pi} / 2$, re- 
spectively. The fundamental mode $\left(\mathrm{TE}_{0}\right)$ is injected from Port 1 and imaged in Port3 and Port6, then extracted from Port9. In addition, the first-order mode $\left(\mathrm{TE}_{1}\right)$ will be spilt into two fundamental modes in the Port2 and Port4 with the same power and $\pi$ phase difference. After transmitting through the tapered $\pi / 2$ phase shifter and MMI2, the two modes will be converted into the $\mathrm{TE}_{0}$ mode through constructive interference in MMI2, and then output from Port8. Therefore, the $\mathrm{TE}_{0}$ mode will be directed out from Port 9 while the $\mathrm{TE}_{1}$ mode will be converted into $\mathrm{TE}_{0}$ mode and coupled out from Port8, thus realizing the mode converter and demultiplexer.

In the design above, an SOI wafer with a $220 \mathrm{~nm}$ top silicon layer and $2 \mu \mathrm{m}$ buried oxide (BOX) layer is considered as the fabrication platform, and the input width of Port 1 is set to be $1 \mu \mathrm{m}$ so that fundamental mode and first order mode can exist at the same time. The width of MMI1 is chosen to be $2.4 \mu \mathrm{m}$ in consideration of the compact device size and fabrication requirement. Firstly, the effective reflective index of the fundamental mode in MMI1 at the wavelength of $1550 \mathrm{~nm}$ is numerically calculated to be $\sim 2.83$, and the length of the MMI1 is $10.5 \mu \mathrm{m}$ according to Equation (1). As shown in Figure 2(a) that the optimal length of MMI1 is $9.2 \mu \mathrm{m}$ when $\mathrm{TE}_{0}$ and $\mathrm{TE}_{1}$ mode injected into Port1 and analyze the power in Port2, Port3 and Port4 respectively, and produce a high transmission

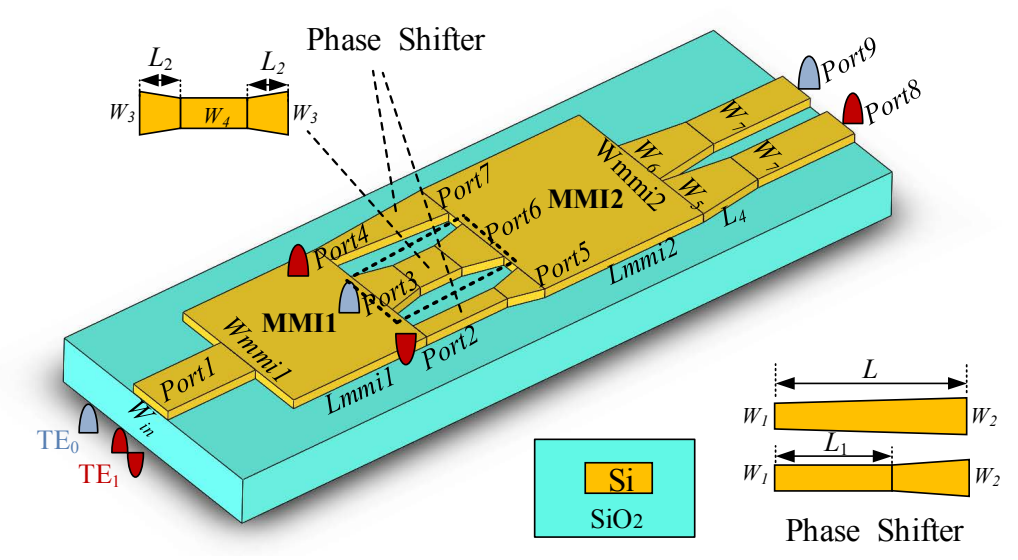

Figure 1. Schematic diagram of the mode converter and demultiplexer structure.
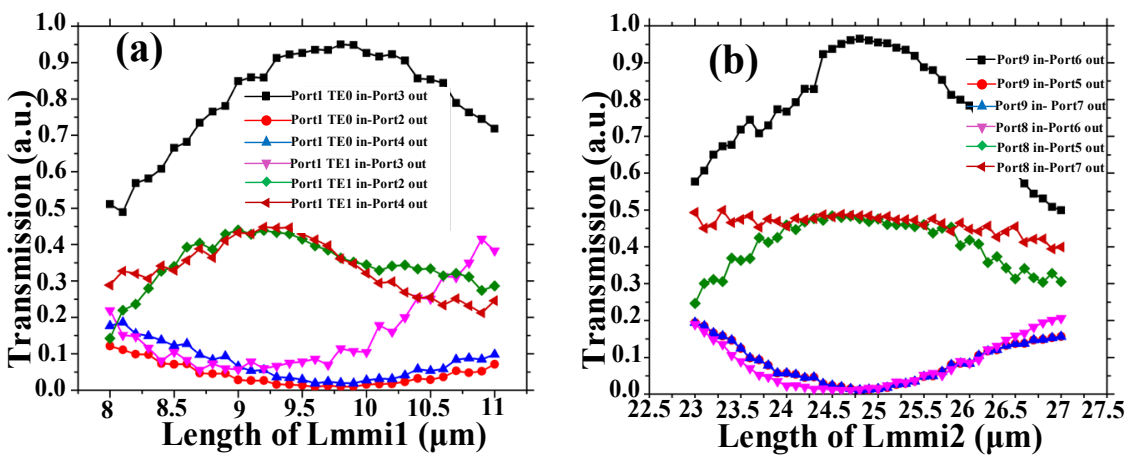

Figure 2. The transmit efficiency varies with (a) the MMI1 length, (b) the MMI2 length. 
efficiency for both $\mathrm{TE}_{0}$ and $\mathrm{TE}_{1}$ modes. For the $3 \mathrm{~dB}$ coupler, MMI2 has a width of $2.7 \mu \mathrm{m}$. Similarly, as TE0 mode injected in Port8 and Port9 and analyzing the power in Port5, Port6 and Port 7 , respectively, the optimal length of MMI2 is 25 $\mu \mathrm{m}$ through FDTD simulations, as exhibited in Figure 2(b).

In order to obtain high transmission efficiency as much as possible, we design and optimize the width of $W_{1}, W_{3}, W_{5}$ and $W_{6}$. As shown in Figure 3(a), we fix the width and length of MMI1, then injected $\mathrm{TE}_{1}$ mode from Port 1 and calculated the power in Port2 (the same power in Port2 and Port4) with the change of the width of $W_{1}$. From the figure we can find that the transmission loss can be reduced to minimum in the case of $W_{1}=0.5 \mu \mathrm{m}$. Similarly, we injected TE0 mode in Port 1 and analyzed the transmission efficiency varied with the width of $W_{3}$, as shown in Figure 3(b), the width is optimized as $W_{3}=0.9 \mu \mathrm{m}$. In order to avoid the coupling between Port2, Port3 and Port4, we set the width of $W_{4}$ and $W_{7}$ as $0.5 \mu \mathrm{m}$, which can only support the fundamental mode, and the length of $L_{2}$ and $L_{4}$ is set as $2 \mu \mathrm{m}$ and $5 \mu \mathrm{m}$. In order to optimize the widths of $W_{5}$ and $W_{6}$, we fix the size of MMI1 and MMI2, then inject $\mathrm{TE}_{0}$ and $\mathrm{TE}_{1}$ mode from Port 1 and change the widths of $W_{5}$ and $W_{6}$ from $0.3 \mu \mathrm{m} 1.3 \mu \mathrm{m}$, respectively. As shown in Figure 3(c) and Figure 3(d), we find the maximum transmission power in Port9 and Port8 in the case of $W_{5}=0.9 \mu \mathrm{m}$ and $W_{6}=0.9 \mu \mathrm{m}$ respectively.

When designing the tapered phase shifter, the length of $L$ is chosen as $9.5 \mu \mathrm{m}$ and the width of $W_{2}$ is $0.8 \mu \mathrm{m}$. Then we scan the phase shift of tapered phase shifter of different length $L 1$, as shown in Figure 4 . The result shown a $\pi / 2$ phase shifter at $1550 \mathrm{~nm}$ with the length of $L_{1}=7.6 \mu \mathrm{m}$. Moreover, we get the phase difference with different wavelength of $1530 \mathrm{~nm}, 1550 \mathrm{~nm}$ and $1570 \mathrm{~nm}$ in Figure 4,
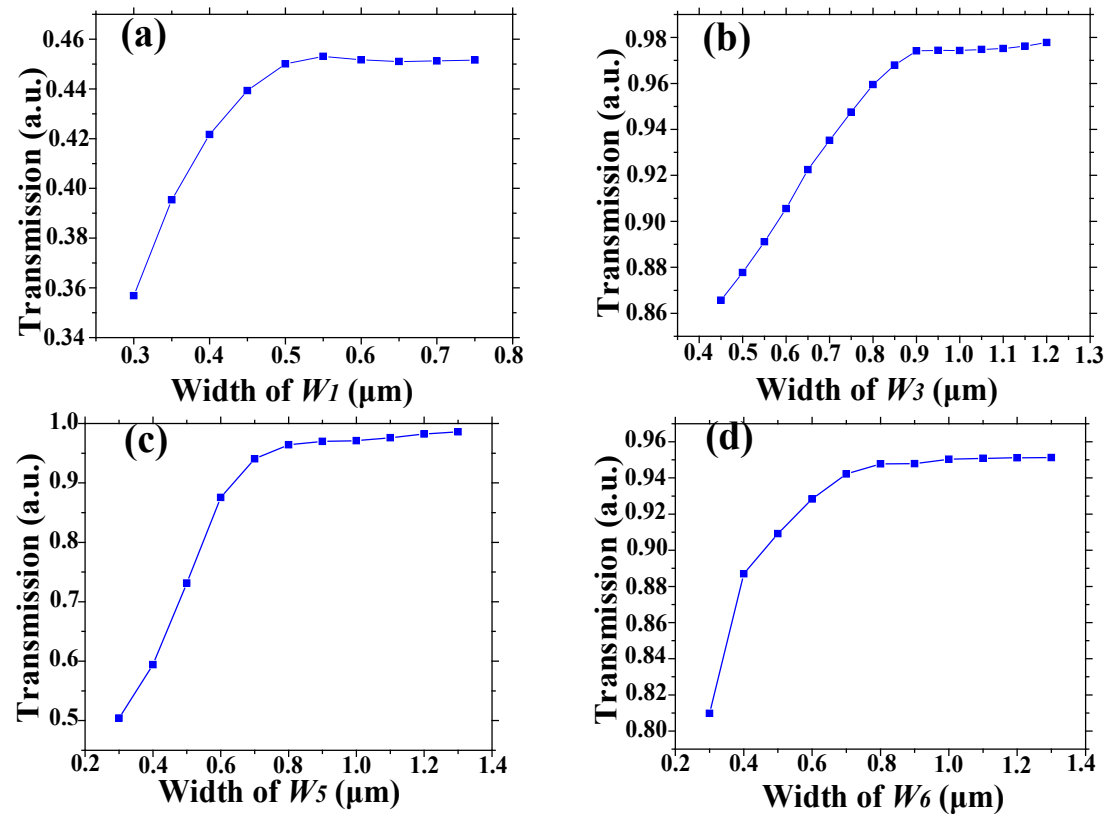

Figure 3. The transmit efficiency varies with the width of (a) $W_{1}$, (b) $W_{2}$, (c) $W_{5}$, (d) $W_{6}$ at $1550 \mathrm{~nm}$. 
which indicates a variation range of lower than two degrees difference range over the whole $\mathrm{C}$ band wavelength (1530 $\mathrm{nm}$ to $1570 \mathrm{~nm}$ ).

Then we calculate the phase difference when $\mathrm{TE}_{0}$ mode injects from Port8 then transmits through MMI2 and phase shifter with wavelength varying from $1530 \mathrm{~nm}$ to $1570 \mathrm{~nm}$, as shown in Figure 5(a). The results clearly show that the phase shift induced by phase shifter is inversely proportional to the wavelength shift, which means a reduction of phase shift when wavelength increases. Nevertheless, the phase difference caused by MMI2 becomes larger with the increase of wavelength. Furthermore, we also analyze the total phase difference of these two components, as exhibited in Figure 5(b). It is obvious that the total phase difference is between -0.025 and 0.025 rad when wavelength varying within $C$ band. Such small phase difference may enhance the transmission performance of the device.

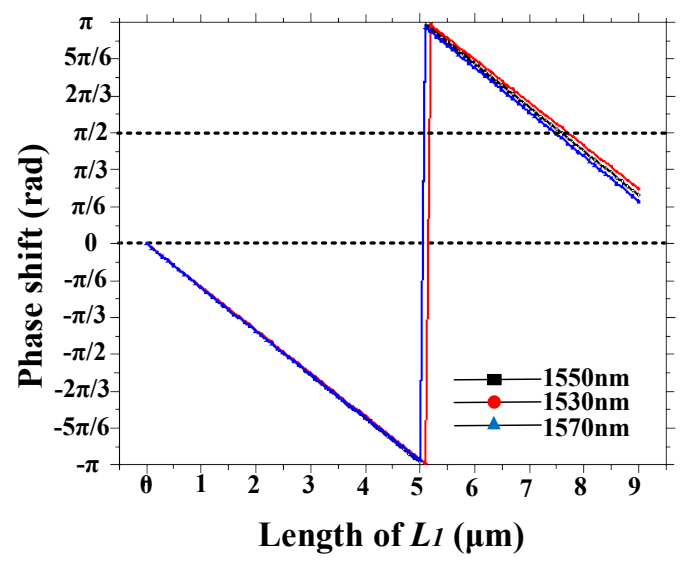

Figure 4. The phase difference of $1530 \mathrm{~nm}, 1550 \mathrm{~nm}$ and $1570 \mathrm{~nm}$ as function of length $L_{1}$.

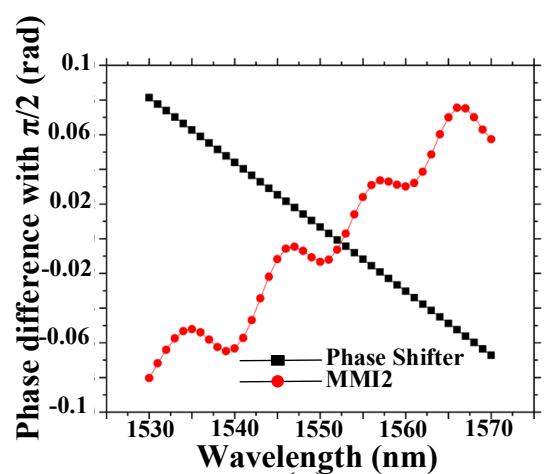

(a)

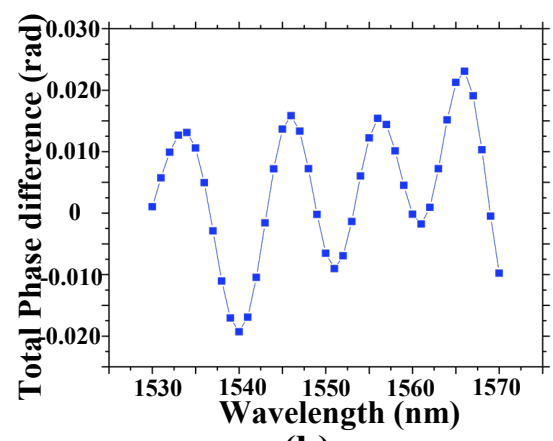

(b)

Figure 5. (a) The phase difference of phase shifter and MMI2 as function of wavelength, (b) the total phase difference of MMI2 and phase shifter as function of wavelength.

\section{Results and Discussion}

The total device is simulated by way of 3D FDTD method. To demonstrate the 
mode converter and multiplexer of $\mathrm{TE}_{0}-\mathrm{TE}_{0}, \mathrm{TE}_{0}-\mathrm{TE}_{1}$, we simulate the optical field distributions, and the results are shown in Figure 6(a) and Figure 6(d). The $\mathrm{TE}_{0}$ mode injected in center-port (Port9 in Figure 1) will keep $\mathrm{TE}_{0}$ mode transmission to the output port. However, the $\mathrm{TE}_{0}$ mode injected in under-port (Port8 in Figure 1) will be converted into $\mathrm{TE}_{1}$ mode with high conversion efficiency. Additionally, for the mode demultiplexer, the light output from Port9 for the $\mathrm{TE}_{0}$ mode input, and the light output from Port8 for $\mathrm{TE}_{1}$ mode input. One should note that both output modes are $\mathrm{TE}_{0}$ modes, as displayed in Figure $6(\mathrm{~b})$ and Figure 6(e). Then we simulate the transmission field distributions of the mode multiplex and de/multiplex structure, as shown in Figure 6(c) and Figure 6(f), there is an obvious and direct correspondence between the input port and the output port.

For a multiplexer and de/multiplexer structure, the two most important parameters are insertion loss (IL) and crosstalk (CT). Here we analyze the relationship between transmission efficiency and wavelength by calculated the IL and CT of the device. Figure 7 shows the result of two modes multiplex, demultiplex and mode multiplex and de/multiplex. Numerical calculation results show

(a)
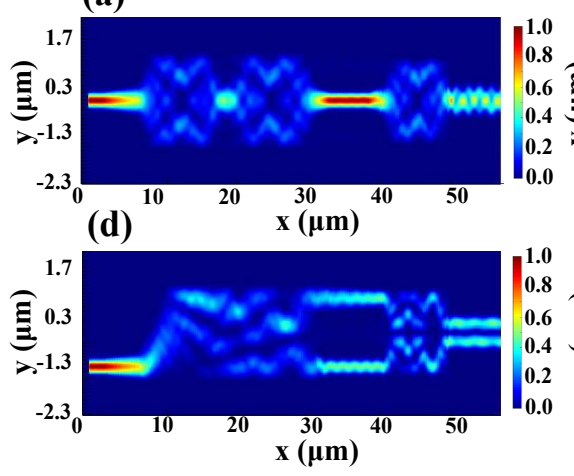

(b)

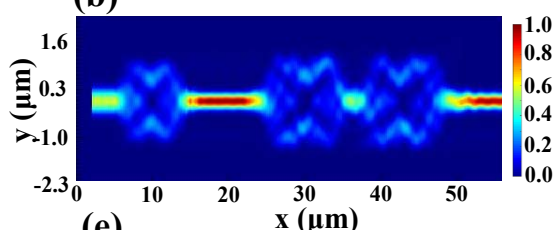

(e) (c)

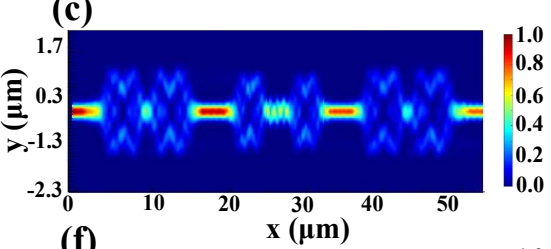

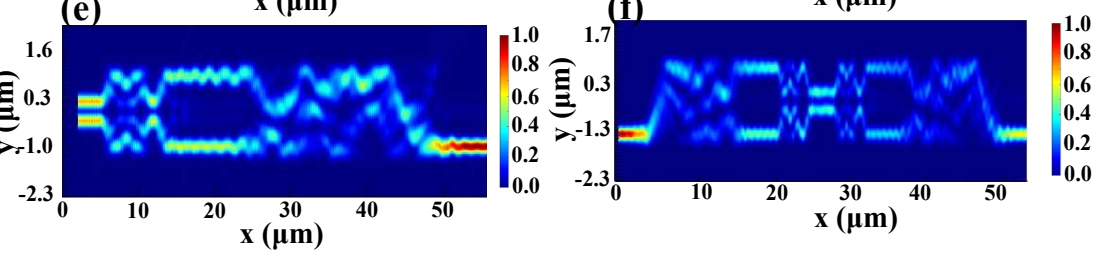

Figure 6. Transmission field distributions of (a), (d) the mode multiplexer with $\mathrm{TE}_{0}$ injected in Port9 and Port8, respectively. (b), (e) the mode demultiplexer structure with $\mathrm{TE}_{0}$ and $\mathrm{TE}_{1}$ injected in Port1, and (c), (f) the mode multiplexer and de/multiplexer with $\mathrm{TE}_{0}$ injected in Port9 and Port8, respectively.

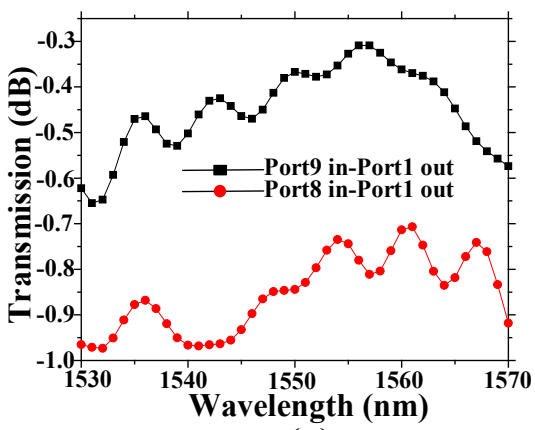

(a)

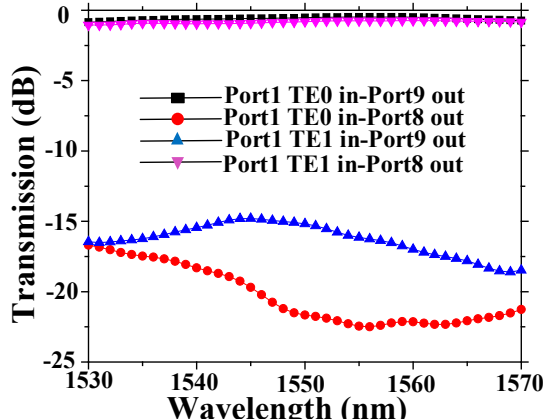

(b)

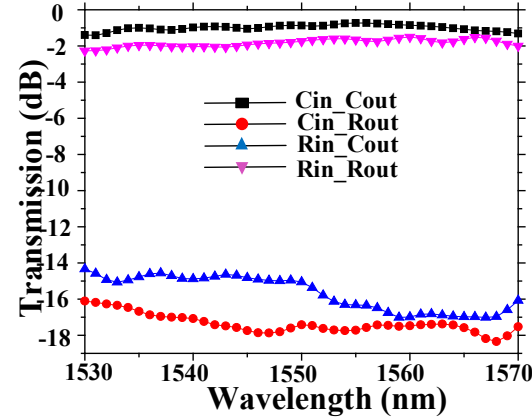

(c)

Figure 7. Wavelength dependence of (a) the mode multiplexer when the input is the $\mathrm{TE}_{0}$ in Port 9 and Port8, (b) the mode demultiplexer structure with $\mathrm{TE}_{0}$ and $\mathrm{TE}_{1}$ injected in Portl, and (c) the mode multiplexer and de/multiplexer when the input is the $\mathrm{TE}_{0}$ in Port9 and Port8, respectively. 
the IL are below $1 \mathrm{~dB}$ in multiplex and de/multiplex structure over the whole $\mathrm{C}$ band ( $1530 \mathrm{~nm}$ to $1570 \mathrm{~nm}$ ), while the IL of the whole structure of multiplex and de/multiplex is below $2 \mathrm{~dB}$ as shown in Figure $7(\mathbf{c})$. It can be seen that the CT of mode multiplexer and de/multiplexer structure is lower than $-14 \mathrm{~dB}$ and the designed two-mode multiplexer is below $-15 \mathrm{~dB}$ within the bandwidth from 1530 $\mathrm{nm}$ to $1570 \mathrm{~nm}$. Such simulated results show that the proposed structure is potential to be applied in practical silicon photonic integrated circuits.

At last, we calculate the fabrication tolerance of the proposed structure. It is clear that there is a quadratic curve relation between the beat length of MMI and the effective width of MMI, in other words, the performance of the device is much sensitive to the width of MMI. Therefore, we set the two mode demultiplexer structure with $\mathrm{TE}_{0}$ and $\mathrm{TE}_{1}$ mode injected into Port1, respectively. Then we analyze the normalized power with the MMI width varying from $-50 \mathrm{~nm}$ to 50 $\mathrm{nm}$ at the wavelength of $1530 \mathrm{~nm}, 1550 \mathrm{~nm}$ and $1570 \mathrm{~nm}$, respectively. As illustrated in Figure 8(a), when MMI width varies from $-10 \mathrm{~nm}$ to $10 \mathrm{~nm}$, the insertion loss is lower than $1.5 \mathrm{~dB}$.

For the phase shifter, its performance highly sensitive to dimensional variations, we simulate the transmission characteristics when the total length varies at the wavelength of $1530 \mathrm{~nm}, 1550 \mathrm{~nm}$ and $1570 \mathrm{~nm}$. It can be easily observed from Figure 8(b) that the maximum transmission occurs when $\Delta L$ is $0 \mathrm{~nm}$ at $1550 \mathrm{~nm}$, and the transmission efficiency decreases whether the $\Delta L$ increases or decreases. In the practical fabrication craft, the length variation can be selected from $-30 \mathrm{~nm}$ to $30 \mathrm{~nm}$, which can provide much convenience.
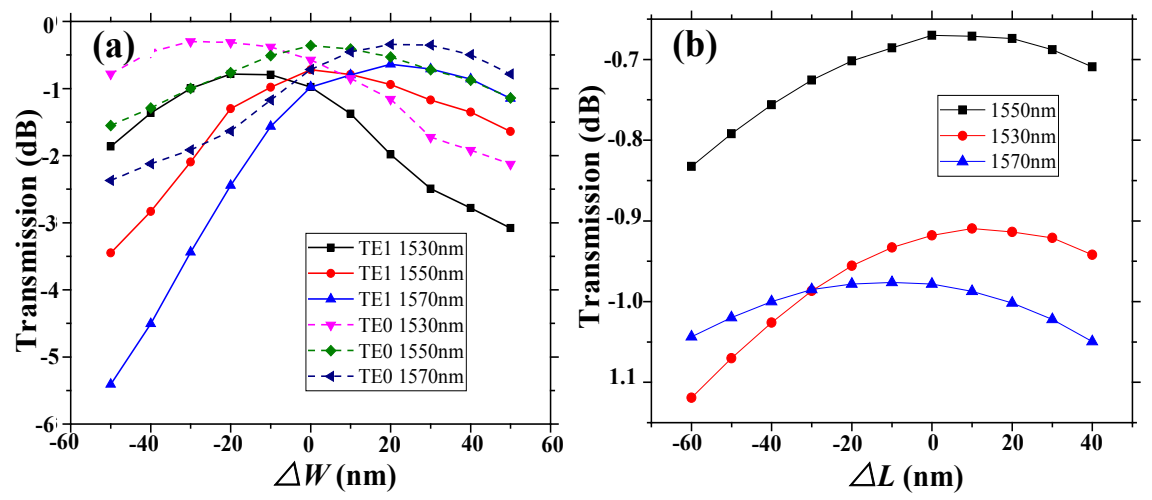

Figure 8. Fabrication tolerance of the (a) transmission efficiency varies with MMI width, (b) transmission efficiency varies with phase shifter length at the wavelength of $1530 \mathrm{~nm}$, $1550 \mathrm{~nm}$ and $1570 \mathrm{~nm}$.

\section{Conclusion}

We propose and simulate a SOI-based mode converter and demultiplexer structure for WDM optical interconnection and transmission by employing MMI and tapered phase shifter structure. The simulation results indicate that such a structure has a broad bandwidth of over the whole $\mathrm{C}$ band, a low transmission loss of less than $1 \mathrm{~dB}$ and a low crosstalk of less than $-15 \mathrm{~dB}$. A compact mode 
converter and demultiplexer of less than $2.7 \mu \mathrm{m} \times 43.7 \mu \mathrm{m}$ is achieved and the fabrication tolerance is $\sim 10 \mathrm{~nm}$ which can meet the demand of the practical fabrication. Such structure may provide a valuable selection in silicon photonic integrated circuits (PICs).

\section{Acknowledgements}

This work is supported by the National Natural Science Foundation of China (NSFC) (Grant No. 61875063).

\section{Conflicts of Interest}

The authors declare no conflicts of interest regarding the publication of this paper.

\section{References}

[1] Li, C., Liu, D. and Dai, D. (2018) Multimode Silicon Photonics. Nanophotonics, 8, 227-247. https://doi.org/10.1515/nanoph-2018-0161

[2] Dai, D., Wang, J., Chen, S., Wang, S. and He, S. (2015) Monolithically Integrated 64-Channel Silicon Hybrid Demultiplexer Enabling Simultaneous Wavelength and Mode Division Multiplexing. Laser and Photonics Reviews, 9, 339-344. https://doi.org/10.1002/lpor.201400446

[3] Xing, J., Li, Z., Xiao, X., Yu, J. and Yu, Y. (2013) Two-Mode Multiplexer and Demultiplexer Based on Adiabatic Couplers. Optics Letters, 38, 3468-3470. https://doi.org/10.1364/OL.38.003468

[4] Driscoll, J.B., Grote, R.R., Souhan, B., Dadap, J.I., Lu, M. and Osgood, R.M. (2013) Asymmetric Y Junctions in Silicon Waveguides for On-Chip Mode-Division Multiplexing. Optics Letters, 38, 1854-1856. https://doi.org/10.1364/OL.38.001854

[5] Chen, W., Wang, P. and Yang, J. (2013) Mode Multi/Demultiplexer Based on Cascaded Asymmetric Y-Junctions. Optics Express, 21, 25113-25119. https://doi.org/10.1364/OE.21.025113

[6] Han, L., Liang, S., Zhu, H., Qiao, L., Xu, J. and Wang, W. (2015) Two-Mode De/Multiplexer Based on Multimode Interference Couplers with a Tilted Joint as Phase Shifter. Optics Letters, 40, 518-521. https://doi.org/10.1364/OL.40.000518

[7] Guo, F., Lu, D., Zhang, R., Wang, H., Liu, S., Sun, M., Kan, Q. and Ji, C. (2016) An MMI-Based Mode (DE) MUX by Varying the Waveguide Thickness of the Phase Shifter. IEEE Photonics Technology Letters, 28, 2443-2446. https://doi.org/10.1109/LPT.2016.2599934

[8] Soldano, L.B. and Pennings, E.C. (1995) Optical Multi-Mode Interference Devices Based on Self-Imaging: Principles and Applications. Journal of Lightwave Technology, 13, 615-627. https://doi.org/10.1109/50.372474 